\title{
VINCULACIÓN Y TRANSFERENCIA DE CONOCIMIENTOS EN LA UNIVERSIDAD NACIONAL DEL LITORAL
}

WILSON, María Lucrecia ${ }^{1}$, MAMMARELLA, Enrique José2

\section{RESUMEN}

En general, las Universidades Nacionales Argentinas, luego de recuperar su autonomía en 1985, debido a un contexto totalmente desfavorable respecto a la situación del Estado Nacional, comenzaron en los años 90s a llevar adelante políticas de vinculación con su entorno.

Acompañando a las Universidades, las actividades de vinculación tecnológica y transferencia de conocimientos ocuparon el centro de las políticas públicas hacia sector socio-productivo, y se llevaron adelante diversos mecanismos promocionales que tuvieron un fuerte impacto en la generalización de nuevas modalidades.

En este contexto, la Universidad Nacional del Litoral (UNL) ha venido instrumentado, desde hace más de tres décadas, políticas concretas de promoción de la innovación y transferencia de los resultado de la investigación, las cuales tienen por objetivo asegurar su apropiación por parte del tejido económico y social. Esta situación propone con mayor fuerza, la necesidad de generar renovados procesos de vinculación entre la Universidad y las organizaciones del medio socio-productivo y gubernamental.

EL objeto del presente trabajo es analizar la política de vinculación de Universidad Nacional del Litoral, con el fin estudiar el modelo de transferencia aplicado en la región Litoral-Centro del país, como así también dimensionar al grado de alcance que tiene esta política en la región.

\section{INTRODUCCIÓN: Políticas de Vin- culación Tecnológica de la UNL}

La Universidad Nacional del Litoral es una institución pública dedicada a la educación superior, la investigación científica, el desarrollo tecnológico y la extensión de sus actividades al medio, incluyendo la transferencia de conocimientos a la sociedad en general y de tecnología al sistema productivo. Desde sus inicios ha contribuido con la sociedad, brindando enseñanza de calidad, buscando y creando nuevos conocimientos y volcándolos al medio a

\footnotetext{
${ }^{1}$ Especialista en Gestión de la Investigación y de la Transferencia de Conocimiento, Universidad Politécnica de Valencia, INGENIO, España. Facultad de Ingeniería Química - Universidad Nacional del Litoral Santiago del Estero 2829 - Santa Fe, Argentina - mlwilson@unl.edu.ar - 0342154398418

${ }^{2}$ Doctor en Ingeniería Química, Universidad Nacional del Litoral. Facultad de Ingeniería Química - Universidad Nacional del Litoral. Santiago del Estero 2829 - Santa Fe, Argentina - ejoma@unl.edu.ar .
} 
través de diversas producciones culturales, científicas y técnicas y formando profesionales con destacadas actuaciones en la vida pública, social, artística y productiva regional. La creación de la UNL, en 1919, como universidad nacional, fue un corolario de de un proceso que comenzó sobre la base de la Universidad Provincial de Santa Fe, que había iniciado sus actividades en 1889.

Actualmente, sus diez facultades e institutos que se encuentran distribuidos en cuatro ciudades: Santa Fe, Esperanza, Reconquista y Gálvez, todas de la provincia de Santa Fe. Estos espacios conforman un ambiente propicio para la formación de 45.114 estudiantes y el trabajo de 3.357 docentes, de los cuales más de la mitad desarrollan tareas de investigación, extensión y transferencia. En esta casa de estudios se brindan 31 carreras de pre grado, 49 carreras de grado y 79 carreras de postgrado.

Teniendo en cuenta que las capacidades científicas y técnicas son consideradas la principal fuente de riqueza en una sociedad moderna y pueden definirse como las potencialidades adquiridas por profesionales altamente calificados, desarrolladas en organizaciones generadoras de conocimientos y experiencias capaces de vincularlas con necesidades y convertirlas en una eventual utilización aplicada.

En este sentido, la UNL ha venido instrumentado, desde hace más de tres décadas, políticas concretas de promoción de la innovación y transferencia de los resultado de la investigación, las cuales tienen por objetivo asegurar su apropiación por parte del tejido económico y social. Esta situación propone con mayor fuerza, la necesidad de generar renovados procesos de vinculación entre la Universidad y las organizaciones del medio socio-productivo y gubernamental. Para implementar políticas de ciencia, tecnología e innovación y contar con estrategias y metodologías que aseguren una adecuada vinculación con los sectores productivos y de servicios es fundamental contar con una unidad de interfaz que contenga:

- Recursos humanos capacitados específicamente para abordar la temática

- Inversión de recursos financieros en la estructuración de una unidad operativa.

- Capacidad de llevar adelante aspectos relacionados con la vinculación, sobre la utilidad de los resultados, el beneficio de la innovación, cuestiones jurídico legales y económicos de los acuerdos, la propiedad intelectual, etc.

\section{Medición de la relación de la UNL con su entorno}

La medición confiable de la innovación es fundamental en el diseño de políticas públicas.Esta acción ayuda a los responsables de elaborar políticas a evaluar su eficiencia y la del gasto que representan, así como a calcular la contribución de esta actividad para alcanzar objetivos sociales y económicos.

En este sentido y dado que los gobiernos nacionales y locales deben basar sus decisiones de asignación de recursos con bases técnicas, es necesario contar con un sistema de indicadores que sustenten estas políticas y ayuden a evaluar el resultado de las mismas.

De la misma manera en la Universidad Nacional del Litoral debe desarrollar y/o adoptar indicadores para medir su desempeño interno y externo, ya que 
se hace necesario contar con un sistema de indicadores de vinculación tecnológica que demuestre la evolución de diferentes actividades llevadas adelante por la Universidad, como así también, que brinde una herramienta de consulta para adoptar nuevas políticas de gestión en la materia.

\section{Selección de indicadores.}

La selección de indicadores fue realizada en base al "Manual de Indicadores de Vinculación de la universidad con el entorno socioeconómico": un marco para la discusión; de Pablo D'Este; Elena Castro Martínez; Jordi MolasGallart. Mayo de 2009. OEI - AECID.

Los indicadores que se estudiarán son aquellos con los que se cuenta la información relevada y sistematizada en el Informe Institucional de la Universidad Nacional del Litoral del año 2013. El período de estudio comprende los años 2009, 2010, 2011, 2012 y 2013. nuación:

Los mismos se presentan a conti-

1. Presencia de la vinculación en las prioridades políticas de la UNL

2. Dedicación de recursos

3. Dotación de estructuras para la vinculación

4. Procesos y documentación de actividades de vinculación

5. Comercialización de tecnología

6. Emprendimientos

7. Asesoramiento y consultoría

8. Contratos de $\mathrm{I}+\mathrm{D}$
9. Prácticas en organizaciones no académicas

10. Cursos y actividades de formación

\section{Aplicación de los indicadores}

1. Presencia de la vinculación en las prioridades políticas de la UNL

Plan de Desarrollo Institucional 20002009

La Universidad instrumentó el Plan de Desarrollo Institucional 2000-2009 sobre la base de los procesos de evaluación institucional (interna y externa) que permitieron elaborar diagnósticos, señalar debilidades, fortalezas, vacancias y potencialidades. Se articuló sobre seis ejes estratégicos en torno a Gobierno, Estudios, Investigación y Desarrollo, Vinculación Tecnológica, Vida Universitaria y Extensión Social y Cultural.

- Una Universidad en la búsqueda permanente de ampliar las fronteras del conocimiento en un adecuado equilibrio entre la investigación fundamental y la orientada hacia objetivos especificos para beneficio de toda la sociedad.

- Una Universidad que interactúe con el sector productivo y el Estado, generando el ambiente propicio para los procesos de innovación cientifica y tecnológica necesarios para el desarrollo sustentable de la región.

Plan de Desarrollo Institucional 20102019: "Hacia la Universidad del Bicentenario"

Hacia finales de 2010, los Órganos Colegiados de Gobierno aprobaron el Plan de Desarrollo Institucional 2010-2019 "Hacia la Universidad del Centenario". Este documento provee los lineamientos políticos institucionales para el planeamiento y la 
gestión en todas las Áreas, Unidades Académicas, Escuelas e Institutos dependientes de la Universidad; siendo éste el segundo ejercicio de planeamiento para el desarrollo (precedido por el PDI 2000-2009).

- Cooperación prioritaria con la innovación en el entorno y conexión con una amplia red de internacionalización.

Estatuto de la Universidad Nacional del $\underline{\text { Litoral }}$

Aprobado en Sesión Extraordinaria de la Asamblea Universitaria 4 de octubre de 2012 Resolución A.U. N 04/12, Publicado en el Boletín Oficial $\mathrm{N}^{\circ} 32.609$ de fecha 27 de marzo de 2013, ordenado por Resolución $\mathrm{N}^{\circ}$ 480/2013 del Ministerio de Educación.

"Articulo 2": Corresponde a la Universidad en el marco del principio constitucional del desarrollo bumano, promover los valores democráticos y la igualdad de oportunidades y posibilidades, con inclusión y sin discriminación alguna; proveer al avance del conocimien- to en todas las disciplinas, cultivándolo en la enseñanza, la investigación y desarrollo y la extensión; y asumir el compromiso con el desarrollo de la Nación, en conexión con el mundo y mediante la cooperación con el Estado y el tejido social, cultural y productivo de su área de influencia, con epicentro en la ciudad de Santa Fe..."

Organigrama de la Universidad Nacional del Litoral y Facultad de Ingeniería Química.

El objetivo de presentar la estructura de la Universidad en tres de sus componentes es mostrar cómo interactúan entre ellos de manera bidireccional respecto a las políticas de vinculación tecnológica que se llevan adelante.

La Universidad cuenta con la Secretaría de Vinculación Tecnológica y Desarrollo Productivo, en la unidad central y por ejemplo, con la Secretaría de Relaciones con el Medio en la Facultad de Ingeniería Química.

2. Dedicación de recursos

\begin{tabular}{|c|c|c|c|c|c|c|}
\hline & & 2009 & 2010 & 2011 & 2012 & 2013 \\
\hline \multirow{6}{*}{$\begin{array}{l}\text { Dedicación } \\
\text { de recursos }\end{array}$} & $\begin{array}{l}\text { Proyectos de investigación } \\
\text { (\$) }\end{array}$ & $19.351 .573,32$ & $12.266 .917,91$ & $13.015 .485,59$ & $8.346 .668,00$ & $8.179 .625,00$ \\
\hline & Programas de extensión (\$) & $1.329 .000,00$ & $2.088 .000,00$ & $2.088 .000,00$ & $2.600 .000,00$ & $3.140 .000,00$ \\
\hline & $\begin{array}{l}\text { Estimulos para } \\
\text { participación en } \\
\text { actividades de vinculación } \\
\text { (\$) }\end{array}$ & $5.969 .387,12$ & $7.797 .865,88$ & $9.467 .364,19$ & $10.840 .019,93$ & $13.500 .386,86$ \\
\hline & $\begin{array}{l}\text { Proyectos de transferencia } \\
\text { de conocimientos(S) }\end{array}$ & $1.232 .226,20$ & $1.440 .747,40$ & $1.567 .224,50$ & $1.941 .213,60$ & $2.392 .756,50$ \\
\hline & $\begin{array}{l}\text { Creación de empresas de } \\
\text { Base Tecnológicas (EBT) } \\
\text { (\$) }\end{array}$ & $580.000,00$ & $707.500,00$ & $1.230 .000,00$ & $1.230 .000,00$ & $1.480 .000,00$ \\
\hline & $\begin{array}{l}\text { Divulgación social de la } \\
\text { ciencia(\$) }\end{array}$ & $50.000,00$ & $50.000,00$ & $40.000,00$ & $92.058,80$ & $158.270,63$ \\
\hline
\end{tabular}




\section{Dotación de estructuras}

\begin{tabular}{|c|c|c|c|c|c|c|}
\hline & & 2009 & 2010 & 2011 & 2012 & 2013 \\
\hline \multirow{9}{*}{$\begin{array}{l}\text { Dotación de estructuras } \\
\text { para la... }\end{array}$} & Gestión de la investigación & si & si & si & si & si \\
\hline & Gestión de los programas de extensión & si & si & si & si & si \\
\hline & Gestión de la Vinculación tecnológica & si & si & si & si & si \\
\hline & Parques cientificos o polos & si & si & si & si & si \\
\hline & Parque tecnológico o polo & si & si & si & si & si \\
\hline & Incubadora de empresas & si & si & si & si & si \\
\hline & Cultura cientifica & si & si & si & si & si \\
\hline & Editorial & si & si & si & si & si \\
\hline & Nuevas formas de innovación & si & si & si & si & si \\
\hline
\end{tabular}

4. Procesos y documentación de actividades de vinculación

Procesos y documentación de actividades de vinculación

\begin{tabular}{|c|c|c|c|c|c|}
\hline & 2009 & 2010 & 2011 & 2012 & 2013 \\
\hline Evaluación de la investigación & no & no & no & no & si \\
\hline Evaluación de las actividades de vinculación & no & no & no & no & no \\
\hline Gestión de la propiedad intelectual & si & si & si & si & si \\
\hline Implicación de becarios en actividades de I+D & si & si & si & si & si \\
\hline $\begin{array}{l}\text { Contratos de I+D con empresas y otras entidades } \\
\text { sociales }\end{array}$ & si & si & si & si & si \\
\hline $\begin{array}{l}\text { Creación de empresas de Base Tecnológicas } \\
\text { (EBT) }\end{array}$ & no & no & no & no & no \\
\hline $\begin{array}{l}\text { Prácticas de estudiantes en empresas y otras } \\
\text { entidades }\end{array}$ & si & si & si & si & si \\
\hline Programas de extensión & si & si & si & si & si \\
\hline Actividades de divulgación científica & no & no & no & no & no \\
\hline
\end{tabular}

5. Comercialización de la Tecnología

\begin{tabular}{|l|l|c|c|c|c|c|}
\cline { 3 - 7 } \multicolumn{2}{c|}{} & $\mathbf{2 0 0 9}$ & $\mathbf{2 0 1 0}$ & $\mathbf{2 0 1 1}$ & $\mathbf{2 0 1 2}$ & $\mathbf{2 0 1 3}$ \\
\hline \multirow{4}{*}{$\begin{array}{l}\text { Comercialización de } \\
\text { tecnologia }\end{array}$} & $\mathrm{N}^{\circ}$ de patentes solicitadas & 8 & 6 & 16 & 8 & 7 \\
\cline { 2 - 7 } & $\mathrm{N}^{\circ}$ de patentes coneedidas & 0 & 4 & 5 & 2 & 3 \\
\cline { 2 - 7 } & $\begin{array}{l}N^{0} \text { de solieitudes de otros titulos de } \\
\text { Propiedad (variedades de vegetales, } \\
\text { software) }\end{array}$ & 6 & 8 & 6 & 9 & 3 \\
\cline { 2 - 7 } & $\mathrm{N}^{\circ}$ de licencias suscritas & 2 & 2 & 0 & 1 & 0 \\
\cline { 2 - 7 } & Volumen de ingresos por licencias & $2.535,00$ & $25.842,51$ & $13.899,66$ & $15.242,50$ & $2219.732,70$ \\
\hline
\end{tabular}




\section{Emprendimientos}

\begin{tabular}{|l|l|c|c|c|c|c|}
\cline { 2 - 6 } \multicolumn{2}{l|}{} & $\mathbf{2 0 0 9}$ & $\mathbf{2 0 1 0}$ & $\mathbf{2 0 1 1}$ & $\mathbf{2 0 1 2}$ & $\mathbf{2 0 1 3}$ \\
\hline \multirow{4}{*}{ Emprendimientos } & $\mathrm{N}^{\circ}$ de spin-offs y start-up & 2 & 3 & 2 & 3 & 4 \\
\cline { 2 - 6 } & $\mathrm{N}^{\circ}$ de cmpleos creados en los spin-offs y start-up & \multicolumn{2}{|c|}{ No se encuentra la información relevada } \\
\cline { 2 - 5 } & Ingresos generados por los spin-offs y start-up & No se encuentra la información relevada \\
\cline { 2 - 5 } & $\begin{array}{l}\text { No se encuentra la información relevada } \\
\text { No de spin-offs y start-up que operan en la } \\
\text { actualidad }\end{array}$ & No \\
\hline
\end{tabular}

\section{Asesoramiento y consultoría}

\begin{tabular}{|c|c|c|c|c|c|c|}
\hline & \\
\hline & & 2009 & 2010 & 2011 & 2012 & 2013 \\
\hline \multirow{10}{*}{$\begin{array}{l}\text { Asesoramiento } \\
\text { y consultoria }\end{array}$} & $\mathrm{N}^{\circ}$ de contratos de consultoria & 19 & so & 49 & 47 & 36 \\
\hline & $\begin{array}{l}\text { Volumen de ingresos obtenidos } \\
\text { derivados de consultaria }\end{array}$ & $1.227 .445,00$ & $7.360 .260,87$ & $3.945 .002,19$ & $9.699 .638,58$ & $5.979 .878,07$ \\
\hline & $\mathrm{N}^{\circ}$ de consultorias a PyMES & 11 & 12 & 20 & 17 & 14 \\
\hline & $\begin{array}{l}\text { Volumen de ingresos de consultoria } \\
\text { a pymes }\end{array}$ & $480.025,00$ & $732.131,16$ & $1.102 .046,00$ & $1.657 .039,00$ & $1.910 .589,04$ \\
\hline & $\begin{array}{l}\mathrm{N}^{\circ} \text { de consultoria a } \\
\text { administraciones públicas }\end{array}$ & 6 & 24 & 18 & 10 & 10 \\
\hline & $\begin{array}{l}\text { Volumen de ingresos de consultoria } \\
\text { a administraciones públicas }\end{array}$ & $665.900,00$ & $6.118 .769,71$ & $1.620 .178,18$ & $1.535 .000,00$ & $1.396 .308,21$ \\
\hline & $\begin{array}{l}\mathrm{N}^{\circ} \text { de profesores involucrados en } \\
\text { actividades de consultoria y } \\
\text { asistencia técnica }\end{array}$ & \multicolumn{5}{|c|}{ No se encuentra la información relevada } \\
\hline & $\begin{array}{l}\mathrm{N}^{\circ} \text { de profesores que participan en } \\
\text { comités asesores, nacionales e } \\
\text { internacionales }\end{array}$ & \multicolumn{5}{|c|}{ No se encuentra la información relevada } \\
\hline & $\begin{array}{l}\begin{array}{l}N^{\circ} \text { de servicios rutinarios y de } \\
\text { calibración }\end{array} \\
\end{array}$ & 10 & 33 & 40 & 44 & 59 \\
\hline & $\begin{array}{l}\text { Volumen de ingresos de servieios } \\
\text { nutinarios y de calibración }\end{array}$ & $215.198,63$ & $3.913 .805,00$ & $1.979 .957,00$ & $6.517 .160,34$ & $11.192 .962,08$ \\
\hline
\end{tabular}

\section{Contratos de $\mathrm{I}+\mathrm{D}$}

\begin{tabular}{|l|l|c|c|c|c|c|}
\cline { 2 - 6 } \multicolumn{2}{c|}{} & 2009 & $\mathbf{2 0 1 0}$ & $\mathbf{2 0 1 1}$ & $\mathbf{2 0 1 2}$ & $\mathbf{2 0 1 3}$ \\
\hline \multirow{3}{*}{$\begin{array}{l}\text { Contratos de } \\
\text { I+D }\end{array}$} & $\begin{array}{l}\text { Ingresos derivados de } \\
\text { contratos de I+D }\end{array}$ & $1.987 .563,00$ & $41.514 .156,00$ & $16.538 .987,00$ & $4.273 .000,00$ & $19.211 .097,00$ \\
\cline { 2 - 6 } & $\begin{array}{l}\mathrm{N}^{5} \text { de contratos establecidos } \\
\text { con organismos no } \\
\text { académicos }\end{array}$ & 4 & 5 & 6 & 6 & 5 \\
\cline { 2 - 7 } & $\begin{array}{l}\mathrm{N}^{\circ} \text { de profesores que } \\
\text { participan en contratos }\end{array}$ & 5 & 16 & 15 & 7 & 17 \\
\hline
\end{tabular}


9. Prácticas en organizaciones no académicas

\begin{tabular}{|l|l|c|c|c|c|c|}
\cline { 3 - 6 } \multicolumn{2}{c|}{} & 2009 & 2010 & 2011 & 2012 & 2013 \\
\hline \multirow{2}{*}{$\begin{array}{l}\text { Prácticas en } \\
\text { organizaciones } \\
\text { no académicas }\end{array}$} & $\begin{array}{l}\text { No de estudiantes que realizan estancias en } \\
\text { organizaciones no académicas como parte de } \\
\text { su carrera de grado }\end{array}$ & 161 & 341 & 459 & 466 & 471 \\
\cline { 2 - 6 } & $\begin{array}{l}\text { No de cursos y seminarios impartidos por la } \\
\text { Universidad en el que se contempla la } \\
\text { colaboración con entidades no académicas } \\
\text { para articular estancias de estudiantes durante } \\
\text { su formación }\end{array}$ & 1 & 2 & 4 & 5 & 7 \\
\hline
\end{tabular}

10. Cursos y actividades de formación

\begin{tabular}{|l|l|c|c|c|c|c|}
\cline { 3 - 6 } \multicolumn{2}{l|}{} & 2009 & 2010 & 2011 & 2012 & 2013 \\
\hline $\begin{array}{l}\text { Cursos y actividades de } \\
\text { formación }\end{array}$ & $\begin{array}{l}\text { Ingresos por cursos } \\
\text { especializados }\end{array}$ & $96.226,00$ & $918.284,09$ & $823.738,75$ & $974.840,25$ & $1.489 .229,29$ \\
\cline { 2 - 7 } & $N^{\circ}$ de cursos especializados & 10 & 9 & 21 & 12 & 11 \\
\hline
\end{tabular}

\section{CONCLUSIONES FINALES}

La experiencia general de la UNL en actividades asociadas a la producción de conocimientos y la posibilidad de su apropiación por parte de la sociedad y el sistema productivo lleva más de treinta años.

Desde la inclusión de estas políticas en su plan de desarrollo institucional hasta la creación de las estructuras internas de gestión y herramientas de administración que permitan instrumentar estas acciones institucionales.

Como se puede observar del análisis de los indicadores, la Universidad focaliza estas políticas basándose en:

-Implementación de estrategias activas en vinculación y transferencia de conocimientos con el medio socio-productivo, principalmente en la región litoral-centro del país.

- Mantener estas estrategias a través de las distintas gestiones de gobierno de la Universidad por más de treinta años.
- Generarlasestructurasparaimplementar estas políticas, tanto en la misma Universidad como en el entorno con el cual se vincula.

- Ser proactiva en el desarrollo de nuevas soluciones jurídico administrativas para encarar los nuevos desafíos de la actividad, adecuándose a los escenarios que se generan con la evolución de la misma.

- Capacitación constante de recursos humanos especializados en la materia.

- Capitalizar la experiencia adquirida en las etapas previas para volcarla en las nuevas.

Con lo expuesto anteriormente, podemos concluir que la Universidad Nacional del Litoral, transfiere conocimientos a través se consultorías y de transferencia de nkow how, y particularmente con la incorporación de sus graduados al sistema socio-productivo que las contienen, como así también, fomentando la cultura emprendedora, con el objeto de crear de empresas de base tecnológica y productiva innovadoras que fortalezcan y potencien la región. 
Referencias Bibliográficas

D'ESTE, P.; CASTRO MARTINEZ, E.; MOLAS-GALLART, J. "Documento de base para un "Manual de Indicadores de Vinculación de la universidad con el entorno socioeconómico": un marco para la discusión”, INGENIO - UPV, Mayo de 2009.

CASTRO MARTINEZ, E.; MOLASGALLART, J; "Estadísticas e Indicadores de Ciencia e Innovación”, MGCI 2014

ALBORNOZ, M.: "Indicadores de innovación: las dificultades de un concepto en evolución". Revista CTS, no 13, vol. 5, Noviembre de 2009 (pág. 9-25)

ALBORNOZ, M.: "Política Científica y Tecnológica. Una visión desde América Latina”, Revista Iberoamericana de Ciencia, Tecnología, Sociedad e Innovación. Diciembre 2001. Número 1
MATOZO, E; SCACCHI, D; RAMIREZ, $\mathrm{N}$ : "Estrategias de la UNL en políticas para la innovación”. XII Seminario Latino - Iberoamericano de Gestión Tecnológica - ALTEC 2007 - Septiembre 2007

RICYT/OEA/CYTED - COLCIENCIAS/ OCYT.; Manual de Bogotá: Normalización de Indicadores de Innovación Tecnológica en América Latina y el Caribe. Marzo 2001.

WILSON, L; SCACCHI, D "Estrategias en promoción tecnológica de la UNL” - Cartagena de Indias - XIII Seminario LatinoIberoamericano de Gestión Tecnológica - ALTEC Colombia. Noviembre 2009

INFORME INSTITUCIONAL 2013, Programa de información y análisis institucional, Universidad Nacional del Litoral. 